\title{
Downregulation of miR-124 promotes the growth and invasiveness of glioblastoma cells involving upregulation of PPP1R13L
}

\author{
WEI-HUA ZHAO ${ }^{1}$, SHENG-QI WU ${ }^{2}$ and YANG-DE ZHANG ${ }^{1}$ \\ ${ }^{1}$ National Hepatobiliary and Enteric Surgery Research Center, Central South University, Changsha 410008; \\ ${ }^{2}$ The Tumor Hospital of Xiangya Medicine School of Central South University, Changsha 410013, P.R. China
}

Received January 11, 2013; Accepted March 12, 2013

DOI: $10.3892 /$ ijmm.2013.1365

\begin{abstract}
R NA-124 (miR-124) plays an important role in regulating growth, invasiveness, stem-like traits, differentiation and apoptosis of glioblastoma cells. PPP1R3L, an inhibitory member of the apoptosis-stimulating protein of p53 family (IASPP), is also able to affect growth, cell cycle progression, metastasis and apoptosis of various types of cancer. To investigate the regulation of PPP1R13L expression by miR-124 and their effects on proliferation, cell cycle transition and invasion in glioblastoma cells, U251 and U373 glioblastoma cells were transfected with miR-124 mimics, its negative control (NC) or an inhibitor. We found that miR-124 was downregulated in glioblastoma tissues, and inversely regulated PPP1R13L expression in U251 and U373 glioblastoma cells. PPP1R13L was found to be a direct target of miR-124 in glioblastoma cells. Overexpression of miR-124 inhibited proliferation, G1/S transition and invasiveness in glioblastoma cells. miR-124 downregulation-mediated malignant progression of glioblastoma was partly attributed to increased PPP1R13L expression. Consequently, our findings provide a molecular basis for the role of miR-124/PPP1R13L in the progression of human glioblastoma and suggest a novel target for the treatment of glioblastoma.
\end{abstract}

\section{Introduction}

Glioblastoma is the most common and lethal primary malignant brain tumor, with an annual incidence of 4.96 cases $/ 100,000$ individuals, and accounts for approximately $50 \%$ of all gliomas (1). Glioblastoma is also an uncommon type of malignancy characterized by localization in the brain, highly invasive behavior and extremely poor prognosis. Currently, the treatment strategies for glioblastoma

Correspondence to: Professor Yang-De Zhang, National Hepatobiliary and Enteric Surgery Research Center, Central South University, Xiangya Road No. 87, Changsha, Hunan 410008, P.R. China

E-mail: zyd99@189.cn

Key words: glioblastoma, microRNA-124, PP1R13L, growth, invasion mainly include surgery, chemotherapy and radiation therapy; however, the overall median survival time of patients is only $\sim 15$ months (2). To improve the quality of life in patients with glioblastoma, research is focusing on its pathogenesis and novel therapeutic targets such as microRNAs (miRNAs) (3).

miRNAs are a class of small, non-coding, single-stranded RNAs which negatively regulate gene expression at the posttranscriptional level, mainly by binding to the 3'-untranslated region (3'-UTR) of their target mRNAs. Numerous studies have demonstrated that aberrant expression of miRNAs is closely associated with proliferation, invasion, metastasis and prognosis in various types of cancer (4-6). The development of glioblastoma is associated with dysregulation of multiple miRNAs including miR-21 (7), miR-29b, miR-125a (8), miR-137 (9), miR-328 (10), miR-218 (11), miR-124 and others (12). miR-124 is enriched in brain with a crucial role in neural development and has been shown to be downregulated in glioma (13), suggesting it functions as a tumor suppressor in brain tumor progression. Studies have shown that miR-124 regulates growth, invasiveness, stem-like traits, differentiation, apoptosis of glioblastoma cells, and these processes have been correlated to multiple target genes $(3,13-17)$. miRNAs and their target genes may represent promising therapeutic targets for glioblastoma.

PPP1R3L, an inhibitory member of the apoptosis-stimulating protein of p53 family (IASPP), is able to promote apoptosis through negative regulation of p53 or by inhibiting the transcriptional activity of $\mathrm{p} 63 / \mathrm{p} 73$ on promoters of proapoptotic genes independent of p53 (18-20) always upregulated in malignant tumors (21-23). In addition, it is also reported that PPP1R3L is also involved in tumorigenesis, cell growth, cell cycle progression, metastasis and chemoresistance (24-27). In glioblastoma cells, silencing of PPP1R3L leads to cell proliferation inhibition and cell cycle arrest (28). Analysis indicates that PPP1R13L is a theoretical target gene of miR-124. But whether PPP1R13L is a direct target of miR-124 has not been confirmed, particularly in glioblastoma cells.

Therefore, in the present study, we investigated the regulation of PPP1R13L expression by miR-124 and their effects on proliferation, cell cycle transition and invasion in glioblastoma cell lines U251 and U373. Our results may provide data for supporting miR-124/PPP1R13L as novel therapeutic, diagnostic or prognostic tools for glioblastoma. 


\section{Materials and methods}

Tumor tissue sample preparation. A total of 9 patients diagnosed with glioblastoma were recruited. The experimental protocols were approved by the ethics committee of our hospital. RNA or protein samples prepared from the tumor tissues and normal tissues were then subjected to quantitative RT-PCR and western blot analysis.

Cell culture and transfection. Glioblastoma cell lines U251 (p53 mutant) and U373 (p53 mutant) were purchased from the China Center for Type Culture Collection (CCTCC, Wuhan, China) and maintained in RPMI-1640 (HyClone, Logan, UT, USA) containing $10 \%$ fetal bovine serum (FBS) (Hangzhou Sijiqing) in a humidified atmosphere of $5 \% \mathrm{CO}_{2}$ at $37^{\circ} \mathrm{C}$. For functional analysis, cells were transfected with miR-124 negative control (scrambled miRNA control), miR-124 mimics, or miR-124 inhibitor (Ambion), using Lipofectamine ${ }^{\circledR} 2000$ (Invitrogen Life Technologies, Carlsbad, CA, USA) according to the manufacturer's recommendations.

Real-time RT-PCR. Total RNA was extracted from cells with TRIzol reagent (Invitrogen) following the manufacturer's instructions. The relative expression level of miR-124 was determined by quantitative real-time RT-PCR using the mirVana $^{\mathrm{TM}}$ qRT-PCR microRNA Detection kit (Ambion) following the manufacturer's instructions. Specific primer sets for miR-124 and U6 (used as an internal reference) were obtained from Ambion. Expression of PPP1R13L mRNA was detected by real-time RT-PCR using the standard SYBRGreen RT-PCR kit (Takara Bio, Inc., Otsu, Japan) following the manufacturer's instructions. The specific primer pairs are as follows: PPP1R13L (131 bp), sense, 5'-GTGGCACGGGTG TTGGCGGA-3' and antisense, 5'-CGATGGAAGAGGCG GCTGATG-3'; $\beta$-actin (202 bp) as an internal control, sense, 5'-AGGGGCCGGACTCGTCATACT-3' and antisense, 5'-GG CGGCACCACCATGTACCCT-3'. The relative expression of PPP1R13L mRNA or miR-124 was quantified using GraphPad Prism 4.0 software (GraphPad Software, San Diego, CA, USA) and the $2^{-\Delta \Delta C \mathrm{Ct}}$ method (29).

Dual luciferase reporter assay. The 3'-UTR of PPP1R13L (NM_001142502) containing the miRNA-124 binding sites and its corresponding mutated sequence were cloned into psiCHECK2 luciferase reporter vector (Promega) downstream of Renilla luciferase, named 3'-UTR PPP1R13L and 3'-UTR Mut PPP1R13L, respectively. Using Lipofectamine 2000, U251 and U373 cells were co-transfected with the reporter constructs and miR-124 mimics, miR-124 inhibitor, negative control (NC) or negative control inhibitor. Luciferase activity was determined after $48 \mathrm{~h}$ using the Dual-Glo substrate system (Promega) and a Beckman Coulter LD 400 luminometer. Data are presented as the ratio of experimental (Renilla) luciferase to control (Firefly) luciferase.

Cell proliferation assay. U251 and U373 cells transfected with NC, miR-124 mimics or its inhibitor in exponential growth were plated at a final concentration of $2 \times 10^{3}$ cells/well in 96 -well plates. The viability of cells was evaluated by MTT assay after 24, 48, 72 and $96 \mathrm{~h}$ of seeding. The optical density at $570 \mathrm{~nm}$ (OD570) of each well was measured with an ELISA reader (ELX-800 Type; BioTek Instruments, Inc., Winooski, VT, USA).

Colony formation assay. The effect of ectopic expression of miR-124 on the colony formation of U373 and U251 cells was analyzed by colony formation assay. Parent cells, NC, miR-124 mimics or miR-124 inhibitor-transfected cells in a 6 -well plate ( 200 cells/well) were cultured for 2 weeks. The cell colonies were washed, fixed and stained with Giemsa. Individual colonies with more than 50 cells were counted.

Cell cycle analysis by flow cytometry (FCM). The cells were digested and collected after $48 \mathrm{~h}$ post-transfection and washed with PBS twice. The cells were resuspended in PBS and then fixed in $70 \%$ ethanol at $4^{\circ} \mathrm{C}$ for $18 \mathrm{~h}$. The cells were washed with PBS and resuspended in staining solution [50 $\mu \mathrm{g} / \mathrm{ml}$ of propidium iodide (PI), $1 \mathrm{mg} / \mathrm{ml}$ of RNase A, and $0.1 \%$ Triton X-100 in PBS]. The stained cells $\left(1 \times 10^{5}\right)$ were then analyzed with a flow cytometer (Beckman Coulter, Miami, FL, USA).

Cell invasion assay. The cell invasion assay was performed using a cell invasion assay kit (Chemicon International, Temecula, CA, USA) according to the manufacturer's guidelines as described by Aspenström et al (30). Briefly, U251 or U373 cells were placed in the upper compartment of the chambers, and RPMI-1640 containing 10\% FBS was added in the lower chambers. After $24 \mathrm{~h}$ of incubation at $37^{\circ} \mathrm{C}$, cells on the upper face of the membrane were wiped off using a cotton swab and cells on the lower face were fixed, stained and observed under a microscope. The dye on the membrane was then dissolved with $10 \%$ acetic acid, dispensed into 96-well plates $(150 \mu \mathrm{l} /$ well), and the optical density at $570 \mathrm{~nm}$ (OD570) of each well was measured with an ELISA reader (ELX-800 Type).

Western blotting. Cells were lysed in cell lysate and then centrifuged at $12,000 \mathrm{x}$ g for $20 \mathrm{~min}$ at $4^{\circ} \mathrm{C}$. The supernatant was collected and denatured. Proteins were separated in $10 \%$ SDS-PAGE and blotted onto polyvinylidene difluoride (PVDF) membranes. The PVDF membranes were treated with TBST containing $50 \mathrm{~g} / \mathrm{l}$ skimmed milk at room temperature for $4 \mathrm{~h}$, followed by incubation with the primary antibodies, anti-PPP1R13L, anti-MMP-9, anti-MMP-13 and anti- $\beta$-actin (Santa Cruz Biotechnology, Inc., Santa Cruz, CA, USA) respectively, at $37^{\circ} \mathrm{C}$ for $1 \mathrm{~h}$. Membranes were rinsed and incubated for $1 \mathrm{~h}$ with the corresponding peroxidase-conjugated secondary antibodies. Chemiluminescence detection was performed with the ECL kit (Pierce Chemical, Rockford, IL, USA). The amount of the protein of interest, expressed as arbitrary densitometric units, was normalized to the densitometric units of $\beta$-actin.

Statistical analysis. Data are expressed as means \pm SD from at least three separate experiments. Statistical analysis was carried out using SPSS 15.0 software. The difference between two groups was analyzed by the Student's t-test. A value of $\mathrm{P}<0.05$ was considered to indicate a statistically significant result. 


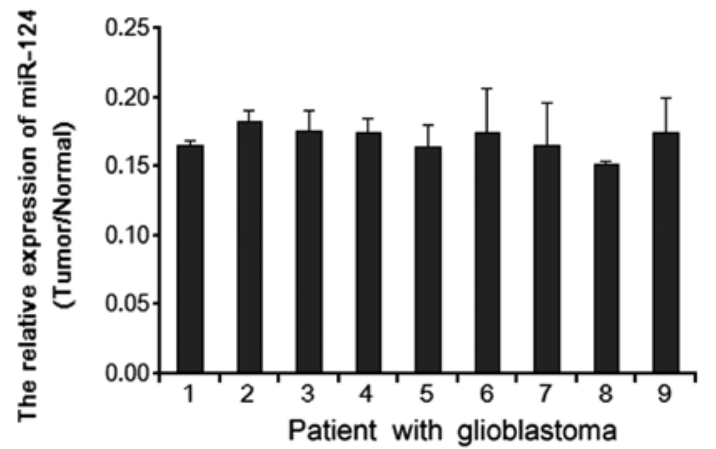

Figure 1. Expression level of miR-124 in glioblastoma tissues relative to adjacent tissues (normal) from the same patient was determined using real-time RT-PCR. Data shown are the means \pm SD of a representative experiment performed in triplicate.

\section{Results}

miR-124 is downregulated in glioblastoma tissues. Real-time quantitative RT-PCR analyses revealed that miR-124 was markedly downregulated in 9 examined tissue samples paired with adjacent nontumor tissues (normal) from the same patient (Fig. 1), indicating that miR-124 is downregulated in human glioblastoma.

miR-124 regulates PPPIR13L expression in glioblastoma cells. miR-124 and PPP1R13L play an important role in cancer development. But whether PPP1R13L is the target gene of miR-124 in glioblastoma cells is unclear. Analysis using available algorithms indicated that PPP1R13L is a theoretical target gene of miR-124 (Fig. 2A). As predicted, ectopic expression of miR-124 in glioblastoma U251 and U373 cells decreased the expression of PPP1R13L mRNA and protein. Consistent with this result, PPP1R13L was upregulated in both types of glioblastoma cells transfected with the miR-124 inhibitor (Fig. 2B-E).

Furthermore, we subcloned the PPP1R13L 3'-untranslated region (3'-UTR) fragment containing the miR-124 binding site and mutated targeting sequence cloned into psi-CHECK2 dual luciferase reporter vectors (named 3'-UTR PPP1R13L and 3'-UTR Mut PPP1R13L, respectively). The result showed that ectopic expression of miR-124 significantly inhibited the luciferase activity in glioblastoma U251 and U373 cells transfected with the 3'-UTR PPP1R13L reporter vector. The

A
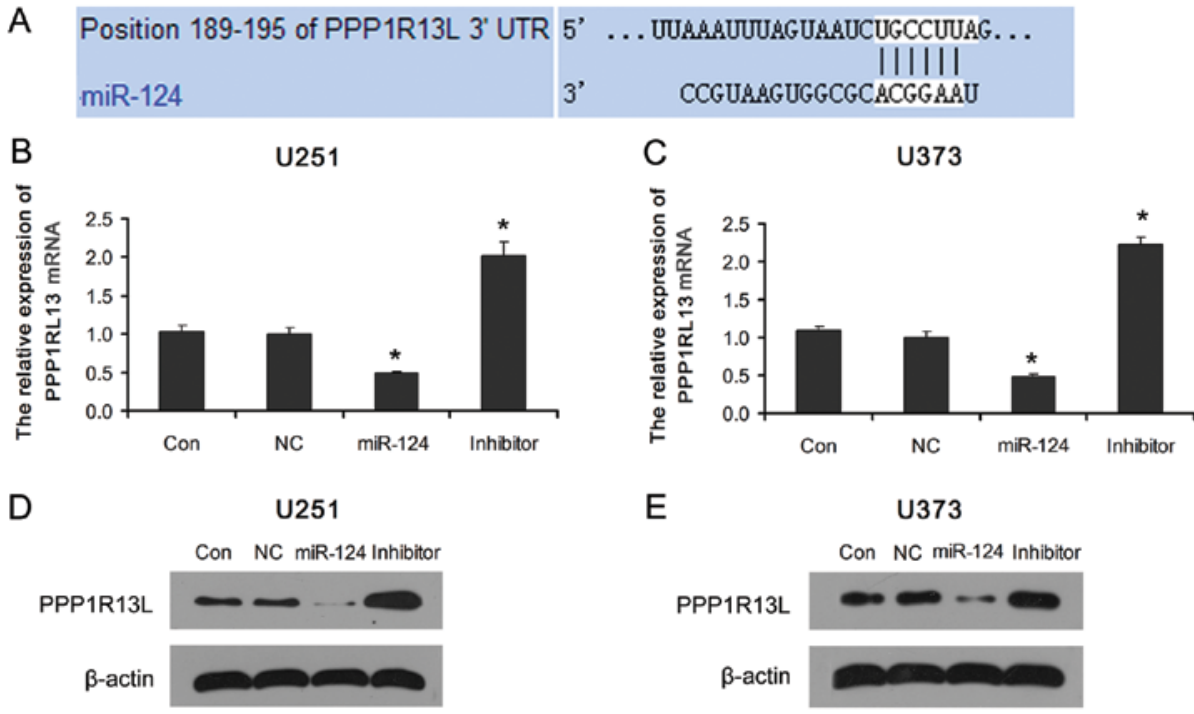

E

U373
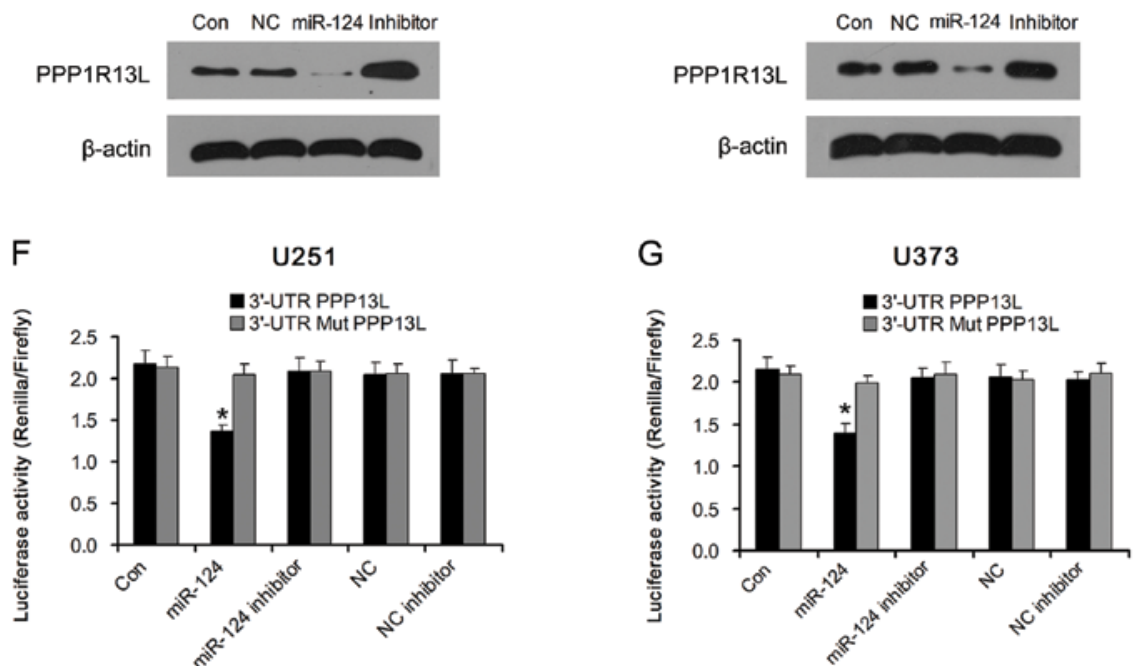

Figure 2. PPP1R13L is a direct target of miR-124. (A) The potential binding site of miR-124 in PPP1R13L 3'-UTR. (B and C) mRNA and (D and E) protein levels of PPP1R13L in glioblastoma U251 and U373 cells transfected with miR-124 mimics, miR-124 inhibtor (Inhibitor) or NC were detected by real-time RT-PCR and western blotting, respectively. ( $F$ and G) Dual luciferase reporter assays were performed to test the interaction of miR-124 and its targeting sequence in the PPP1R13L 3'-UTR, using constructs containing the targeting sequence (named 3'-UTR PPP1R13L) and mutated targeting sequence (named 3'-UTR Mut PPP1R13L) cloned into psi-CHECK2. Data shown are the means \pm SD of a representative experiment performed in triplicate. " $\mathrm{P}<0.05$ vs. NC 
A

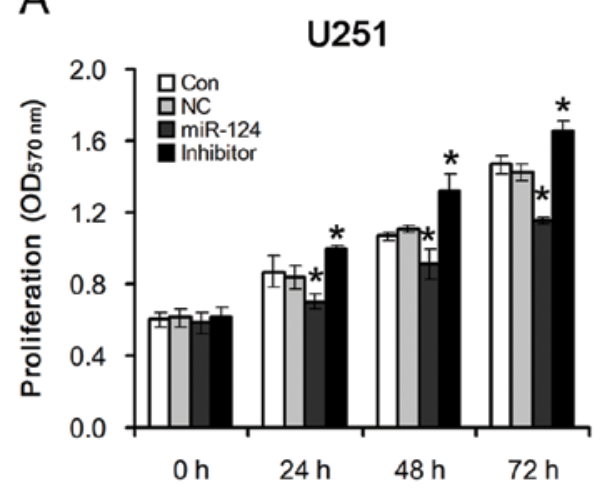

C

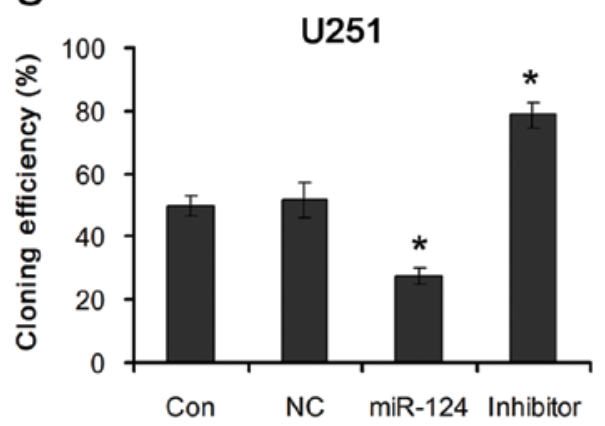

E

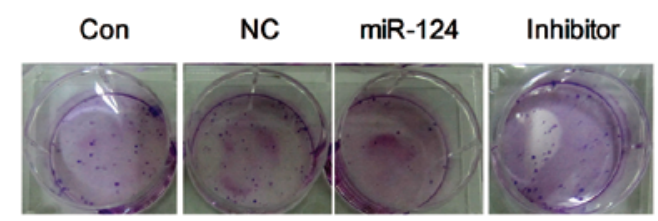

B

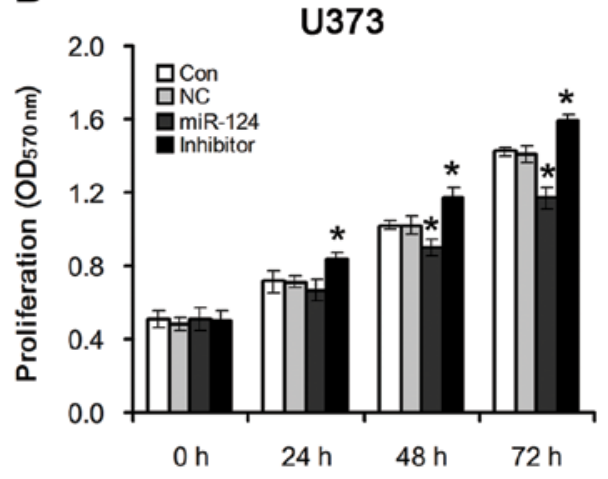

D

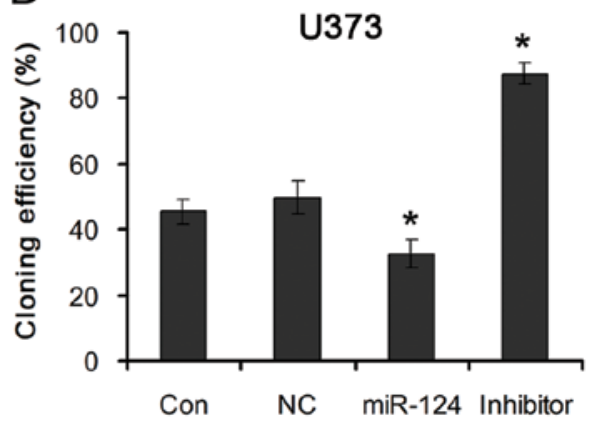

$\mathrm{F} \quad$ U373

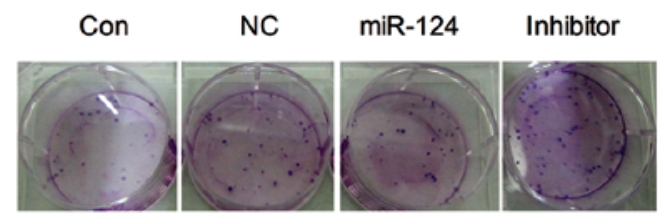

Figure 3. Effects of miR-124/PPP1R13L on cell proliferation and colony formation. The glioblastoma cells were transfected with miR-124 mimics, miR-124 inhibtor (Inhibitor) or NC of miR-124. (A and B) The cell viability from 0 to $72 \mathrm{~h}$ was measured by MTT assay. (C and D) Cloning efficiency was calculated after culturing for 2 weeks. (E and F) Representative results of the colony formation assay. Data shown are the means \pm SD of a representative experiment performed in triplicate. ${ }^{*} \mathrm{P}<0.05$ vs. NC.

luciferase activity levels in glioblastoma U251 and U373 cells transfected with the 3'-UTR Mut PPP1R13L reporter vector or miR-124 inhibitor were restored (Fig. 2F and G). Taken together, our results demonstrated that PPP1R13L is a target of miR-124 and an inverse correlation exists between miR-124 and PPP1R13L in glioblastoma cells.

miR-124-mediated PPPIR13L regulates proliferation of glioblastoma cells. To investigate the effects of miR-124-mediated PPP1R13L on the proliferation of glioblastoma cells, U251 and U373 cells were transfected with miR-124 mimics or its inhibitor. By using MTT and colony formation assays, we observed that overexpression of miR-124 dramatically decreased the growth rate of both types of glioblastoma cells as compared with that of the NC-transfected cells. However, inhibition of miR-124 increased the growth rate of both types of glioblastoma cells as compared with that of the NC-transfected cells (Fig. 3A and B). This suggests that downregulation of miR-124, which results in PPP1R13L upregulation, specifically promotes the proliferation of glioblastoma cells.
miR-124-mediated PPPIR13L regulates G1/S phase transition of glioblastoma cells. To further explore whether miR-124-mediated PPP1R13L increases the proliferation of glioblastoma cells by cell cycle transition, U251 and U373 cells transfected with miR-124 mimics or its inhibitor were analyzed by flow cytometry. The results showed a significant increase in the percentage of cells in the G1/G0 phase and a decrease in the percentage of cells in the $S$ phase in the miR-124-overexpressing cells, and a decrease in G1/G0 phase cells and an increase in S-phase cells in the glioblastoma cells transfected with the miR-124 inhibitor (Fig. 4). The above data suggest that enhancement of glioblastoma cell growth by downregulation of miR-124 may be mediated through regulation of cellular entry into the G1/S transition phase and its target PPP1R13L is possibly implicated in this process.

miR-124-mediated PPPIR13L regulates invasion of glioblastoma cells. To understand the effects of miR-124-mediated PPP1R13L on glioblastoma cell metastasis, the invasiveness of the glioblastoma U251 and U373 cells transfected with miR-124 mimics or its inhibitor was examined. We observed 

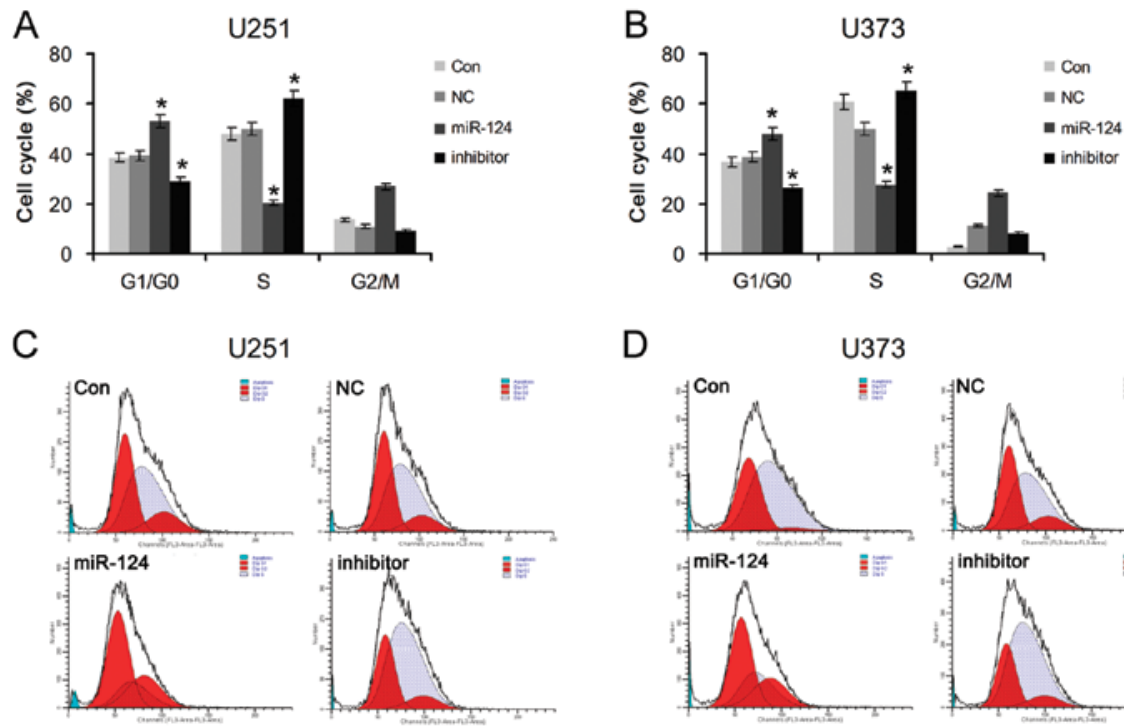

D

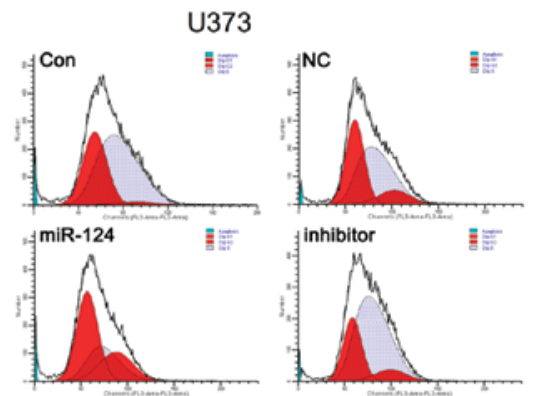

Figure 4. Effects of miR-124/PPP1R13L on cell cycle distribution. (A and B) The cell cycle distribution at $48 \mathrm{~h}$ was determined by propidium iodide (PI) staining and flow cytometric (FCM) analysis. (C and D) Representative FCM results. Data shown are the means \pm SD of a representative experiment performed in triplicate. ${ }^{*} \mathrm{P}<0.05$ vs. NC.

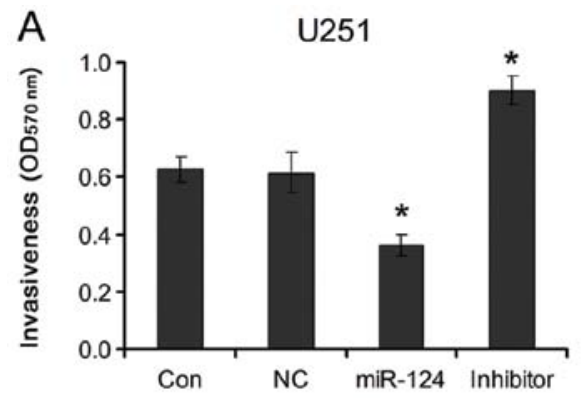

C

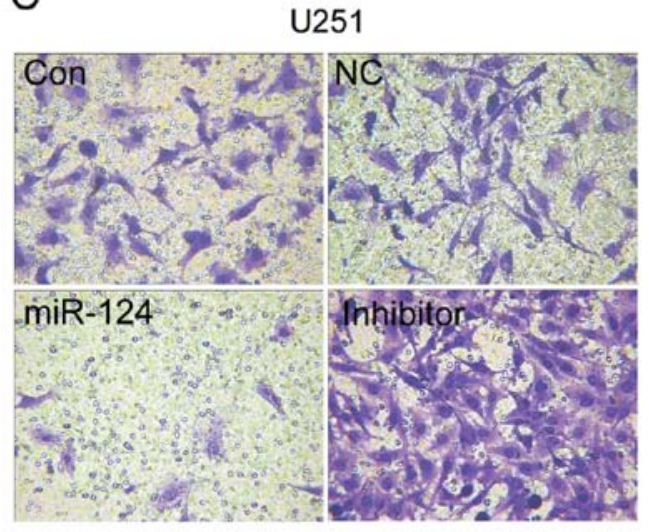

E

$\mathrm{U} 251$

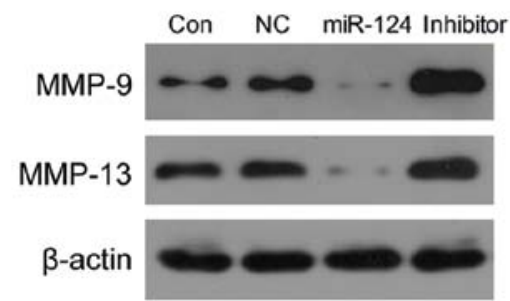

B

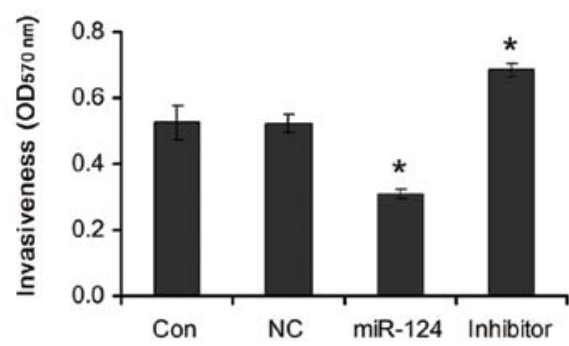

D

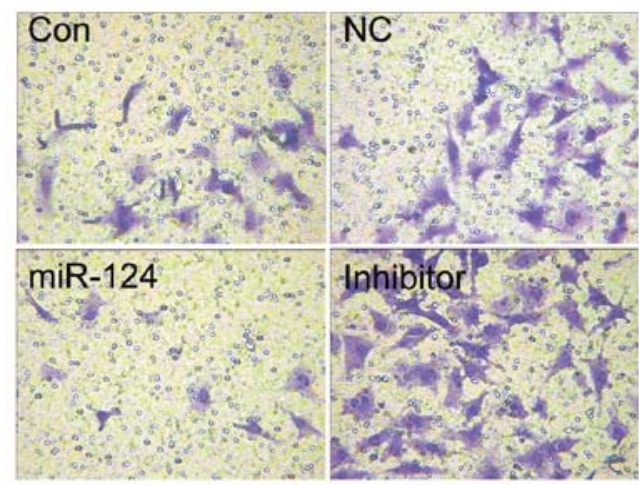

$\mathrm{F}$

U373

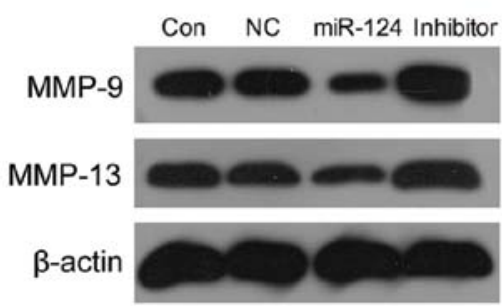

Figure 5. Effects of miR-124/PPP1R13L on cell invasiveness. (A and B) The invasive capabilities were measured by Transwell assay. (C and D) Representative results of the Transwell assay. (E and F) Representative western blotting results indicating MMP-9 and MMP-13 expression in both cell lines. Data shown are the means $\pm \mathrm{SD}$ of a representative experiment performed in triplicate. ${ }^{*} \mathrm{P}<0.05 \mathrm{vs}$. NC. 
that, compared to $\mathrm{NC}$, overexpression of miR-124 in U251 and U373 cells obviously inhibited their invasive ability; however, the invasive ability was increased after inhibition of miR-124 in U251 and U373 cells (Fig. 5A-D). Meanwhile, we found that MMP-9 and MMP-13, molecular markers of cancer metastasis, were decreased after transfection of the cells with miR-124 mimics, and the miR-124 inhibitor increased their expression levels as compared with NC (Fig. 5E and F). This indicates that loss of miR-124 expression mediates PPP1R13L upregulation consequently promoting glioblastoma cell invasion.

\section{Discussion}

miR-124 is known to play an important role in the progression of diverse types of cancers $(5,31)$. In the present study, we found that miR-124 was downregulated in the examined 9 tissue samples, which is consistent with a previous report (12). Our data further illustrate that miR-124 serves as a tumor suppressor in glioblastoma and its abnormal downregulation facilitates tumor progression. It is well known that miRNAs function mainly via specific binding to 3'-UTR of target genes. Theoretic analysis indicated that the 3'-UTR of PPP1R13L includes the binding sites of miR-124. To validate this presumption, glioblastoma U251 and U373 cells were transfected with miR-124 mimics or its inhibitor, and the results showed that the PPP1R13L protein level was accordingly decreased or increased, suggesting that miR-124 negatively regulates PPP1R13L expression in glioblastoma cells and that PPP1R13L is the potential target gene of miR-124. The dual luciferase reporter assay showed that only miR-124 mimics could inhibit the luciferase activity in glioblastoma U251 and U373 cells transfected with the 3'-UTR PPP1R13L reporter vector. Therefore, for the first time, we demonstrated that PPP1R13L is a target gene of miR-124 in glioblastoma cells.

The potential effects of the miR-124/PPP1R13L pathway on glioblastoma U251 and U373 cells was investigated. The results showed that upregulation of miR-124, which led to decreased PPP1R13L expression, inhibited cell proliferation, colony formation, G1/S phase transition and invasive ability of glioblastoma cells. However, downregulation of miR-124 by its inhibitor, which led to increased PPP1R13L expression, promoted these processes. These results suggest that miR-124 downregulation may play a critical role in malignant progression of glioblastoma, and its mechanism of action involves PPP1R13L.

The mechanisms underlying the function of miR-124 as a tumor suppressor in glioma are implicated in the inhibition of proliferation (e.g. via targeting SLC16A1) (13), cell cycle progression (e.g. via targeting CDK6) $(14,15)$, invasiveness (17), differentiation (e.g. by suppressing Twist and SLUG) (16), stem cell characteristics (e.g. via targeting SNAI2, NRAS or PIM3) $(3,17)$. In the present study, several of these molecules or others possibly participated in the process of the effects of miR-124 on the growth and invasive ability of glioblastoma U251 and U373 cells. We believe that PPP1R13L, as a target of miR-124, also plays a certain role in the miR-124-mediated suppression of growth and metastasis of glioblastoma cells. Studies indicate that PPP1R13L is important for tumor cell proliferation. Downregulation of
PPP1R13L leads to growth inhibition in various types of cancers $(25,32,33)$. It has been reported that downregulation of PPP1R13L expression inhibits proliferation and induces cell cycle arrest at G0/G1 phase, which involves the increase in cyclin D1 and a decrease in $\mathrm{p} 21^{\mathrm{waf} 1 / \mathrm{cip} 1}$ expression in U251 cells (28). PPP1R3L is also associated with invasion and lymph node metastasis, as validated in embedded endometrial endometrioid adenocarcinoma (34). Overexpression of PPP1R3L increases the invasiveness of tumor cells through a p53-dependent or p53-independent pathway (35). Our results confirmed that miR-124 negatively regulates PPP1R13L expression in glioblastoma cells with mutant p53. Although miR-124 has numerous target genes, it is logical to deduce that the promotion of cell proliferation, cell cycle progression and invasion in glioblastoma cells by miR-124 downregulation is, at least, partly due to PPP1R13L upregulation.

In conclusion, miR-124 is downregulated in glioblastoma, and negatively regulates PPP1R13L expression in glioblastoma U251 and U373 cells. miR-124 downregulation-mediated malignant progression of glioblastoma is partly attributed to increased PPP1R13L expression. Consequently, our findings provide a molecular basis for the role of miR-124/PPP1R13L in the progression of human glioblastoma cells and suggest a novel target for the treatment of glioblastoma.

\section{References}

1. Baldi I, Huchet A, Bauchet L and Loiseau H: Epidemiology of glioblastoma. Neurochirurgie 56: 433-440, 2010.

2. Preusser M, de Ribaupierre S, Wöhrer A, et al: Current concepts and management of glioblastoma. Ann Neurol 70: 9-21, 2011.

3. Lang MF, Yang S, Zhao C, et al: Genome-wide profiling identified a set of miRNAs that are differentially expressed in glioblastoma stem cells and normal neural stem cells. PLoS One 7: e36248, 2012.

4. Skalsky RL and Cullen BR: Reduced expression of brainenriched microRNAs in glioblastomas permits targeted regulation of a cell death gene. PLoS One 6: e24248, 2011.

5. Sun Y, Zhao X, Zhou Y and Hu Y: miR-124, miR-137 and miR-340 regulate colorectal cancer growth via inhibition of the Warburg effect. Oncol Rep 28: 1346-1352, 2012.

6. Blenkiron C and Miska EA: miRNAs in cancer: approaches, aetiology, diagnostics and therapy. Hum Mol Genet 16: R106-R113, 2007.

7. Li Y, Zhao S, Zhen Y, et al: A miR-21 inhibitor enhances apoptosis and reduces $\mathrm{G}(2)-\mathrm{M}$ accumulation induced by ionizing radiation in human glioblastoma U251 cells. Brain Tumor Pathol 28: 209-214, 2011.

8. Cortez MA, Nicoloso MS, Shimizu M, et al: miR-29b and miR-125a regulate podoplanin and suppress invasion in glioblastoma. Genes Chromosomes Cancer 49: 981-990, 2010.

9. Chen L, Wang X, Wang H, et al: miR-137 is frequently downregulated in glioblastoma and is a negative regulator of Cox-2. Eur J Cancer 48: 3104-3111, 2012.

10. Wu Z, Sun L, Wang H, et al: MiR-328 expression is decreased in high-grade gliomas and is associated with worse survival in primary glioblastoma. PLoS One 7: e47270, 2012.

11. Liu Y, Yan W, Zhang W, et al: MiR-218 reverses high invasiveness of glioblastoma cells by targeting the oncogenic transcription factor LEF1. Oncol Rep 28: 1013-1021, 2012.

12. Li D, Chen P, Li XY, et al: Grade-specific expression profiles of miRNAs/mRNAs and docking study in human grade I-III astrocytomas. OMICS 15: 673-682, 2011.

13. Li KK, Pang JC, Ching AK, et al: miR-124 is frequently downregulated in medulloblastoma and is a negative regulator of SLC16A1. Hum Pathol 40: 1234-1243, 2009.

14. Silber J, Lim DA, Petritsch C, et al: miR-124 and miR-137 inhibit proliferation of glioblastoma multiforme cells and induce differentiation of brain tumor stem cells. BMC Med 6: 14,2008 
15. Pierson J, Hostager B, Fan R and Vibhakar R: Regulation of cyclin-dependent kinase 6 by microRNA 124 in medulloblastoma. J Neurooncol 90: 1-7, 2008.

16. Xie YK, Huo SF, Zhang G, et al: CDA-2 induces cell differentiation through suppressing Twist/SLUG signaling via miR-124 in glioma. J Neurooncol 110: 179-186, 2012.

17. Xia H, Cheung WK, Ng SS, et al: Loss of brain-enriched miR-124 microRNA enhances stem-like traits and invasiveness of glioma cells. J Biol Chem 287: 9962-9971, 2012.

18. Cai Y, Qiu S, Gao X, Gu SZ and Liu ZJ: iASPP inhibits p53-independent apoptosis by inhibiting transcriptional activity of $\mathrm{p} 63 / \mathrm{p} 73$ on promoters of proapoptotic genes. Apoptosis 17: 777-783, 2012.

19. Alsafadi S, Tourpin S, André F, Vassal G and Ahomadegbe JC: P53 family: at the crossroads in cancer therapy. Curr Med Chem 16: 4328-4344, 2009.

20. Bergamaschi D, Samuels Y, Sullivan A, et al: iASPP preferentially binds p53 proline-rich region and modulates apoptotic function of codon 72-polymorphic p53. Nat Genet 38: 1133-1141, 2006.

21. Chen J, Xie F, Zhang L and Jiang WG: iASPP is over-expressed in human non-small cell lung cancer and regulates the proliferation of lung cancer cells through a p53 associated pathway. BMC Cancer 10: 694, 2010.

22. Lu B, Guo H, Zhao J, et al: Increased expression of iASPP regulated by hepatitis $B$ virus $X$ protein-mediated NF- $\kappa B$ activation, in hepatocellular carcinoma. Gastroenterology 139: 2183-2194, 2010.

23. Zhang X, Wang M, Zhou C, Chen S and Wang J: The expression of iASPP in acute leukemias. Leuk Res 29: 179-183, 2005.

24. Pang MS, Chen X, Lu B, et al: Lentiviral vector-mediated doxycycline-inducible iASPP gene targeted RNA interference in hepatocellular carcinoma. Chin J Cancer 29: 796-801, 2010.

25. Lin BL, Xie DY, Xie SB, et al: Down-regulation of iASPP in human hepatocellular carcinoma cells inhibits cell proliferation and tumor growth. Neoplasma 58: 205-210, 2011.
26. Liu Z, Zhang X, Huang D, et al: Elevated expression of iASPP in head and neck squamous cell carcinoma and its clinical significance. Med Oncol 29: 3381-3388, 2012.

27. Jiang L, Siu MK, Wong OG, et al: iASPP and chemoresistance in ovarian cancers: effects on paclitaxel-mediated mitotic catastrophe. Clin Cancer Res 17: 6924-6933, 2011.

28. Li G, Wang R, Gao J, Deng K, Wei J and Wei Y: RNA interference-mediated silencing of iASPP induces cell proliferation inhibition and $\mathrm{G}_{0} / \mathrm{G}_{1}$ cell cycle arrest in U251 human glioblastoma cells. Mol Cell Biochem 350: 193-200, 2011.

29. Arocho A, Chen B, Ladanyi M and Pan Q: Validation of the 2-DeltaDeltaCt calculation as an alternate method of data analysis for quantitative PCR of BCR-ABL P210 transcripts. Diagn Mol Pathol 15: 56-61, 2006.

30. Aspenström P, Fransson A and Saras J: Rho GTPases have diverse effects on the organization of the actin filament system. Biochem J 377: 327-337, 2004.

31. Shi XB, Xue L, Ma AH, et al: Tumor suppressive miR-124 targets androgen receptor and inhibits proliferation of prostate cancer cells. Oncogene: Oct 15, 2012 (Epub ahead of print). doi: 10.1038/onc.2012.425

32. Liu T, Li L, Yang W, et al: iASPP is important for bladder cancer cell proliferation. Oncol Res 19: 125-130, 2011.

33. Zhang B, Xiao HJ, Chen J, Tao X and Cai LH: Inhibitory member of the apoptosis-stimulating protein of p53 (ASPP) family promotes growth and tumorigenesis in human p53-deficient prostate cancer cells. Prostate Cancer Prostatic Dis 14: 219-224, 2011.

34. Liu WK, Jiang XY, Ren JK and Zhang ZX: Expression pattern of the ASPP family members in endometrial endometrioid adenocarcinoma. Onkologie 33: 500-503, 2010.

35. Laska MJ, Lowe SW, Zender L, et al: Enforced expression of PPP1R13L increases tumorigenesis and invasion through p53-dependent and p53-independent mechanisms. Mol Carcinog 48: 832-842, 2009 